\title{
THE STRUCTURE OF SUPERMANIFOLDS ${ }^{1}$ \\ BY
}

\author{
MARJORIE BATCHELOR
}

\begin{abstract}
The increasing recognition of Lie superalgebras and their importance in physics inspired a search to find an object, a "supermanifold", which would realize the geometry implicit in Lie superalgebras. This paper analyzes the structure of supermanifolds as defined by B. Kostant. The result is the following structure theorem.

THE MAN THEOREM. If $E$ is a real vector bundle over the smooth manifold $X$, let $\Lambda E$ be the associated exterior bundle and let $\Gamma(\Lambda E)$ be the sheaf of sections of $\Lambda E$. Then every supermanifold over $X$ is isomorphic to $\Gamma(\Lambda E)$ for some vector bundle $E$ over $X$.

Although the vector bundle $E$ is not unique but is determined only up to isomorphism, and the isomorphism guaranteed is not canonical, the existence of the isomorphism provides a base for a better understanding of geometry in the graded setting.
\end{abstract}

Introduction. The main theorem says that isomorphism classes of vector bundles over $X$ are in one to one correspondence with isomorphism classes of supermanifolds over $X$. The set of isomorphism classes of vector bundles over $X$, and the set of isomorphism classes of supermanifolds over $X$ can each be described as the first Cech cohomology set of $X$ with coefficients in an appropriate sheaf of automorphisms. The proof of the main theorem then depends on translating the problem into cohomological terms describing the structure of the sheaf of automorphisms, and finally computing the cohomology.

Basic definitions and conventions are included in $\S 1$. The structure of the sheaf of automorphisms is discussed in $\$ 2$. The proof of the main theorem is outlined in $\S 3$, and $\S \S 4$ and 5 contain the proofs of lemmas used in $\S \S 2$ and 3.

\section{Preliminaries.}

1.1. Conventions and notation. $X$ will be a smooth manifold. $C^{\infty}$ will be the sheaf of smooth real-valued functions on $X$.

All work will be done over the reals. By a graded algebra $A$ we will mean an algebra $A$ with a $Z_{2}$ grading such that $A=A_{0}+A_{1}$, where if $a$ is in $A_{i}$ and $b$ is in $A_{j}$ then $a b=(-1)^{i j} b a$.

Received by the editors March 6, 1978 and, in revised form, August 1, 1978.

AMS (MOS) subject classifications (1970). Primary 58A05.

${ }^{1}$ This work is contained in the author's doctoral thesis. Thanks are due to Edward Miller for his generous help, encouragement, and infectious enthusiasm. 
1.2. Sheaves and restriction maps. If $Q$ is a sheaf and $V$ and $U$ are open sets in $X$ such that $U<V$ then for $a$ in $Q(V)$, the restriction of $a$ to $\mathbb{Q}(U)$ will be written $\left.a\right|_{U}$ when we wish to emphasize that we are considering $a$ restricted to $\mathbb{Q}(U)$. More often we will simply write $a$ for $\left.a\right|_{U}$. Denote by $\left.Q\right|_{U}$ the sheaf $\mathbb{Q}$ considered as a sheaf over $U$.

1.3. Cohomology. Throughout this paper cohomology means Čech cohomology for sheaves of not necessarily abelian groups. Definitions and properties can be found in [6].

1.4. Let $V$ be a vector space. It will be convenient to have some notation describing various subspaces of the exterior algebra $\Lambda V$. Let $\Lambda^{i} V$ be the subspace of homogeneous elements of degree $i$. Then define

$$
\begin{aligned}
\Lambda V(r) & =\bigoplus_{i>r} \Lambda^{i} V, \\
\Lambda V(o, r) & =\bigoplus_{2 i+1>r} \Lambda^{2 i+1} V, \\
\Lambda V(e, r) & =\bigoplus_{2 i>r} \Lambda^{2 i} V .
\end{aligned}
$$

Now define sheaves over $X$ by setting

$$
\begin{aligned}
\frac{\Lambda V}{V(U)} & =C^{\infty}(U) \otimes \Lambda V, \\
\frac{\Lambda V(r)}{V(U)} & =C^{\infty}(U) \otimes \Lambda V(r), \\
\frac{\Lambda V(o, r)}{\Delta V}(U) & =C^{\infty}(U) \otimes \Lambda V(o, r), \\
\underline{\Lambda V(e, r)}(U) & =C^{\infty}(U) \otimes \Lambda V(e, r)
\end{aligned}
$$

for every open set $U$ in $X$.

Definition 1.5. A supermanifold $(X, A)$ consists of a manifold $X$, called the underlying manifold, and a sheaf of graded algebras $A$ together with a map of sheaves of graded algebras $A \rightarrow C^{\infty}$ where the grading on $C^{\infty}$ is given by $C^{\infty}(U)=C^{\infty}(U)_{0}$ for any open set $U$ in $X$. Moreover the sheaf $A$ must satisfy the local triviality condition: There exists an open cover $\left\{U_{i}\right\}_{I}$ of $X$ and isomorphisms of sheaves of graded algebras $T_{i}:\left.\left.A\right|_{U_{i}} \rightarrow \Lambda R^{n}\right|_{U_{i}}$ for some positive integer $n$. This integer $n$ will be called the odd-dimension of $(X, A)$. We will write $A$ for $(X, A)$ when it is clear which underlying manifold $X$ is intended.

1.6. Morphisms of supermanifolds over $X$. If $A$ and $B$ are supermanifolds over $X$, then a morphism $A \rightarrow B$ over $X$ is a map of the sheaves of graded algebras.

2. Structure theorems for $\operatorname{Aut}\left(\Lambda R^{n}\right)$.

2.1. The local triviality condition makes the task of classifying supermanifolds of fixed odd-dimension $n$ over a fixed underlying manifold $X$ accessible to study by cohomological techniques. Define the sheaf $\operatorname{Aut}\left(\Lambda R^{m}\right)$ of groups 
of automorphisms of $\Lambda R^{n}$ by setting $\operatorname{Aut}\left(\Lambda R^{n}\right)(U)=$ Automorphisms of the sheaf of $Z_{2}$ graded algebras $\left.\left.\Lambda R^{n}\right|_{U}\right\}$ for every open set $U$ in $X$. The following theorem translates the classification problem into a cohomological one.

THEOREM 2.2. There is a bijection between the set $H^{1}\left(X, \operatorname{Aut}\left(\Lambda R^{n}\right)\right)$ and the set of isomorphism classes of supermanifolds over $X$ of odd-dimension $n$.

This is a standard result and the proof will be omitted. See [6, p. 41] for a proof of the analogous result for fiber bundles.

The solution to the cohomology problem depends on an understanding of the sheaf $\operatorname{Aut}\left(\underline{\Lambda R^{n}}\right)$. We have the following structure theorem for $\operatorname{Aut}\left(\underline{\Lambda R^{n}}\right)$.

THEOREM 2.3. Let $\mathcal{C}, \mathcal{D}$ be sheaves of sets over $X$ defined by

$$
\begin{aligned}
& \mathcal{C}(U)=\underline{\operatorname{Der}}(U) \otimes \Lambda R^{n}(e, 2), \\
& \mathscr{D}(U)=\underline{\operatorname{GL}(n)}(U) \times \operatorname{Hom}\left(R^{n}, \underline{\left.\Lambda R^{n}(0,3)\right),}\right.
\end{aligned}
$$

where Der is the sheaf of derivations of $C^{\infty}$ (i.e., sections of the tangent bundle of $X)$ and $\mathrm{GL}(n)$ is the sheaf of invertible $n \times n$ matrices with entries in $C^{\infty}$. Let $Q$ be the sheaf given by $\mathbb{Q}(U)=C(U) \times \mathscr{D}(U)$. Then there is an isomorphism of sheaves of sets $\Phi: \Theta \rightarrow \operatorname{Aut}\left(\Lambda R^{m}\right)$.

The proof will be given in $\$ 5$.

2.4. Using $\Phi$ to identify $\mathbb{Q}$ with $\operatorname{Aut}\left(\Lambda R^{m}\right)$, the essence of this result is converted to a more useful form via the following filtration of $\operatorname{Aut}\left(\Lambda R^{n}\right)$. Define sheaves of groups $\mathbb{Q}_{i}$ for $i=0,1, \cdots, n+1$ by setting $\mathbb{Q}_{i}(U)=\left\{\alpha \in \operatorname{Aut}\left(\underline{\Lambda R^{n}}\right)(U):\right.$

$$
\left.\alpha(x)-x \in \underline{\Lambda R^{n}(i)}(U) \text { for all } x \in \underline{\Lambda R^{n}}(U)\right\}
$$

for every open set $U$ in $X$. This gives a filtration of sheaves:

$$
\text { Id }=\mathbb{Q}_{n+1} \leqslant \mathbb{Q}_{n} \leqslant \cdots \leqslant \mathbb{Q}_{2} \leqslant \mathbb{Q}_{1} \leqslant \mathbb{Q}_{0}=\operatorname{Aut}\left(\underline{\Lambda R^{n}}\right) .
$$

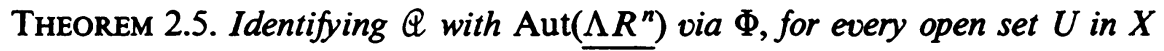
we have

(a) $\mathbb{Q}_{i}(U)$ is a normal subgroup of $\mathbb{Q}(U)$ for $i=0, \ldots, n+1$;

(b)

$$
\mathbb{Q}_{i}(U) \cong\left\{\begin{array}{l}
\operatorname{Der}(U) \otimes \Lambda R^{n}(e, i) \times \operatorname{Hom}\left(R^{n}, \underline{\Lambda R^{n}(o, i)}\right), \text { for } i>2, \\
\mathbb{Q}(U), \text { for } i=0,1 ;
\end{array}\right.
$$

(c)

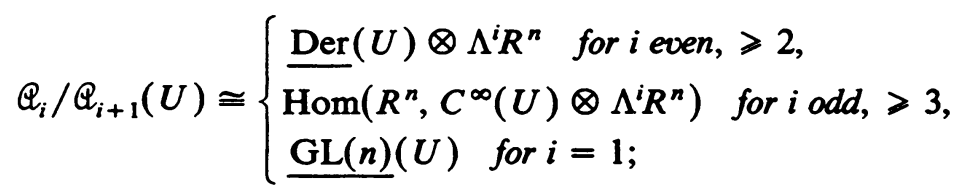


(d) Using (b) we can define an action of $C^{\infty}(U)$ on $Q_{i}(U)$ for $i=0, \ldots, n$ which coincides with the standard action of $C^{\infty}(U)$ on the quotients $Q_{i} / Q_{i+1}(U)$ under the identifications in (c). Moreover, if $z$ is an automorphism of $\mathbb{Q}_{i} / \mathbb{Q}_{i+1}(U)$ arising from conjugation by an element in $\mathcal{Q}(U)$, then $z$ is an automorphism of a $C^{\infty}(U)$ module.

The proof will be given in $\S 5$.

3. Restatement and proof of the main theorem. The main theorem can be restated in a form more convenient for proof.

THEOREM 3.1. The map of sheaves $p: \mathbb{Q} \rightarrow \mathbb{Q} / \mathbb{Q}_{2} \cong \mathrm{GL}(n)$ induces an isomorphism on cohomology $p_{*}: H^{1}(X, \mathbb{Q}) \rightarrow H^{1}\left(X, \mathbb{Q} / \mathbb{Q}_{2}\right)=\bar{H}^{1}(X, \underline{\mathrm{GL}}(n))$.

REMARK 3.2. Theorem 3.1 is equivalent to the main theorem. By Theorem 2.2, $H^{1}(X, \mathbb{Q})$ is in one-one correspondence with the set of isomorphism classes of supermanifolds of odd-dimension $n$. But also $H^{1}(X, \mathrm{GL}(n))$ is in

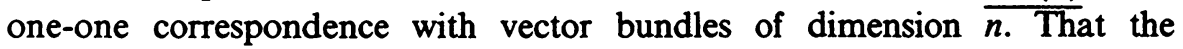
correspondence associates a vector bundle $E$ to the class of the supermanifold $(X, \Gamma(\Lambda E))$ will be shown in establishing the surjectivity of $p_{*}$.

ProOF OF THEOREM 3.1:

Surjectivity of $p_{*}$. Let $h$ in $H^{1}\left(X, \mathbb{Q} / Q_{2}\right)$ be represented by a cocycle $\left\{h_{i j}\right\}$ with respect to some open cover $\left\{U_{i}\right\}_{I}$ of $X$. Then $h_{i j}$ is in $Q / Q_{2}\left(U_{i} \cap U_{j}\right)$ which can be identified with $\operatorname{GL}(n)\left(U_{i} \cap U_{j}\right)$. Using this identification, since $\mathbb{Q}=e \times \mathscr{D}$, there is an obvious choice for coset representative for $h_{i j}$ in $\mathcal{Q}\left(U_{i} \cap U_{j}\right)$, namely $\left(0,\left(h_{i j}, 0\right)\right)$. The collection $\left\{\left(0,\left(h_{i j}, 0\right)\right)\right\}$ is a cocycle and the class it represents in $H^{1}(X, \mathbb{Q})$ is sent to $h$ under $p_{*}$. Effectively, if $E$ is the vector bundle corresponding to the cocycle $\left\{h_{i j}\right\}$ then $\left\{\left(0,\left(h_{i j}, 0\right)\right)\right\}$ is a cocycle corresponding to the supermanifold $\Gamma(\Lambda E)$.

Injectivity of $p_{*} \cdot p_{*}$ is fitted into an exact sequence using the following lemma, which is stated in its general form since it will be used in other contexts later.

LEMMA 3.4. If $\mathcal{G}$ is a sheaf of groups on $X$ and $\mathcal{H}$ is a sheaf of subgroups such that for every open set $U$ in $X, \mathcal{H}(U)$ is a normal subgroup of $\mathcal{G}(U)$, then the sequence of sheaves of groups Id $\rightarrow \mathcal{H} \rightarrow \mathcal{G} \rightarrow \mathcal{G} / \mathcal{H} \rightarrow$ Id gives rise to an exact sequence of pointed sets $H^{1}(X, \mathcal{H}) \rightarrow H^{1}(X, \mathcal{S}) \rightarrow H^{1}(X, \mathcal{S} / \mathcal{H})$.

The proof is a direct computation and is omitted.

Applying this lemma to the sequence Id $\rightarrow \mathbb{Q}_{2} \rightarrow \mathbb{Q} \rightarrow \mathbb{Q} / \mathbb{Q}_{2} \rightarrow$ Id we see that $p_{*}$ fits into the portion

$$
H^{1}\left(X, \mathbb{Q}_{2}\right) \stackrel{j}{\rightarrow} H^{1}(X, \mathbb{Q}) \stackrel{p_{F}}{\rightarrow} H^{1}\left(X, \mathbb{Q} / \mathbb{Q}_{2}\right) .
$$

Unfortunately this is exact only as pointed sets, and even knowing that 
$H^{1}\left(X, Q_{2}\right)=0$ does not guarantee that $p_{*}$ is injective. This situation is remedied by "twisting" the sheaves $Q, \mathbb{Q}_{2}$ and $\mathbb{Q} / \mathbb{Q}_{2}$ as follows.

Definition 3.5. Let $\left\{V_{j}\right\}_{J}$ be an open cover of $X$ and let $\mathcal{G}$ be a sheaf of groups on $X$. Let $\tau=\left\{\tau_{i j}\right\}$ be a collection where $\tau_{i j}$ is an automorphism of the sheaf $\left.\mathcal{G}\right|_{V_{i} \cap V_{j}}$. Define a new sheaf of groups $\mathcal{G}^{\tau}$ by defining $\mathcal{G}^{\tau}(V)$ for any open set $V$ in $X$ to be the kernel of the map

$$
\begin{aligned}
\prod_{j} \mathcal{G}\left(V \cap V_{j}\right) & \rightarrow \prod_{(h, k)} \mathcal{G}\left(V \cap V_{h} \cap V_{k}\right) \\
\left(g_{j}\right) & \mapsto\left(\tau_{k h}\left(g_{h}^{-1}\right) \cdot g_{k}\right) .
\end{aligned}
$$

EXAMPLE 3.6. Let $\mathcal{G}=\mathbb{Q}_{r}$. Let $z=\left\{z_{i j}\right\}$ be a cocycle with respect to a cover $\left\{V_{j}\right\}_{j}$ with values in $\mathcal{Q}$. Then since $\mathbb{Q}_{r}\left(V_{i} \cap V_{j}\right)$ is normal in $\mathbb{Q}\left(V_{i} \cap\right.$ $\left.V_{j}\right)$, conjugation by $z_{i j}, a \mapsto z_{i j} a z_{i j}^{-1}$ for $a$ in $Q_{r}\left(V_{i} \cap V_{j}\right)$ is an automorphism of $\left.\mathbb{Q}_{r}\right|_{V_{i} \cap V_{j}}$. Write $\mathbb{Q}_{r}^{z}$ for the sheaf $\mathbb{Q}$ twisted by conjugation by $z$. If $\bar{z}=\left\{\bar{z}_{i j}\right\}$ is the image of the cocycle $z$ under the projection $p$, we can define the sheaf $Q / \mathbb{Q}_{2}^{z}$ to be the sheaf $Q / Q_{2}$ twisted by $\bar{z}$ in a similar manner.

The following lemmas and a diagram chase complete the proof of Theorem 2.2.

LEMMA 3.7. $H^{1}\left(X, Q_{2}^{z}\right)=0$ for all cocycles $z$ representing any class in $H^{1}(X, \mathbb{Q})$.

LEMMA 3.8. Let $z$ be a cocycle representing a class in $H^{1}(X, Q)$. Then $p_{*}$ fits into the following commutative diagram

$$
\begin{aligned}
& H^{1}(X, \mathbb{Q}) \stackrel{p_{\rightarrow}}{\rightarrow} H^{1}\left(X, \mathbb{Q} / \mathbb{Q}_{2}\right) \\
& \sigma_{\mathbf{z}} \downarrow \mathbb{R} \quad \mathbb{R} \downarrow \rho_{\mathbf{z}} \\
& H^{1}\left(X, \mathbb{Q}_{2}^{z}\right) \stackrel{j^{z}}{\rightarrow} H^{1}\left(X, \mathbb{Q}^{z}\right) \stackrel{p_{Z}^{z}}{\rightarrow} H^{1}\left(X, \mathbb{Q} / \mathbb{Q}_{2}^{z}\right)
\end{aligned}
$$

where the bottom row is an exact sequence of pointed sets and $\sigma_{z}, \rho_{z}$, are bijections, and $\sigma_{z}[z]$ is the basepoint of $H^{1}\left(X, \mathbb{Q}^{z}\right)$.

The proof is completed by the following diagram chase. If $v$ and $w$ represent classes $[v]$ and $[w]$ in $H^{1}(X, \mathbb{Q})$ such that $p_{*}[v]=p_{*}[w]$, consider the diagram (3.9) twisted by $w$ (instead of $z$ ). Then $p_{*}^{w} \sigma_{w}[w]=p_{*}^{w} \sigma_{w}[v]$ is the basepoint of $H^{1}\left(X, \mathbb{Q} / \mathbb{Q}_{2}^{w}\right)$. Since the bottom row is exact as a sequence of pointed sets, there exist $a, b$ in $H^{1}\left(X, \mathbb{Q}_{2}^{w}\right)$ such that $j^{w} a=\sigma_{w}[w]$ and $j^{w} b=$ $\sigma_{w}[v]$. But since $H^{1}\left(X, \mathbb{Q}_{2}^{w}\right)$ has only one element, $a=b$, and $\sigma_{w}[w]=\sigma_{w}[v]$. Since $\sigma_{w}$ is an isomorphism, $[w]=[v]$.

\section{Proofs of Lemmas 3.7, 3.8.}

Proof OF LEMMA 3.7. Notice that the filtration Id $=\mathbb{Q}_{n+1}<\mathbb{Q}_{n}<\cdots<$ $\mathbb{Q}_{2}<\mathbb{Q}$ induces a filtration on $\mathbb{Q}^{z}: \mathrm{Id}=\mathbb{Q}_{n+1}^{z}<\mathbb{Q}_{n}^{z}<\cdots \mathbb{Q}_{2}^{z}<\mathbb{Q}^{z}$. Each $\mathbb{Q}_{r}^{z}$ is still normal in $\mathbb{Q}^{z}$. Since conjugation by $z_{i j}$ is an automorphism of 
$\mathbb{Q}_{r} / \mathbb{Q}_{r+1}^{z}\left(V_{i} \cap V_{j}\right)$ as $C^{\infty}\left(V_{i} \cap V_{j}\right)$ modules, $\mathbb{Q}_{r} / \mathbb{Q}_{r+1}^{z}(U)$ inherits a $C^{\infty}(U)$ module structure (where $U$ is any open set in $X$ ). Consider the following diagram

$$
\begin{aligned}
& H^{1}\left(X, \mathbb{Q}_{n}^{z}\right) \stackrel{j_{n}}{\rightarrow} H^{1}\left(X, \mathbb{Q}_{n-1}^{z}\right) \rightarrow \cdots \rightarrow H^{1}\left(X, \mathbb{Q}_{3}^{z}\right) \quad \stackrel{j_{3}}{\rightarrow} \quad H^{1}\left(X, \mathbb{Q}_{2}^{z}\right) \\
& \begin{array}{ccc}
\downarrow & \downarrow & \downarrow \\
H^{1}\left(X, \mathbb{Q}_{n-1} / \mathbb{Q}_{n}^{z}\right) & H^{1}\left(X, \mathbb{Q}_{3} / \mathbb{Q}_{4}^{z}\right) & H^{1}\left(X, \mathbb{Q}_{2} / \mathbb{Q}_{3}^{z}\right)
\end{array}
\end{aligned}
$$

We know that $H^{1}\left(X, \mathbb{Q}_{r} / \mathbb{Q}_{r+1}^{2}\right)=0$ for $r=2, \ldots, n$, since $\mathbb{Q}_{r} / \mathbb{Q}_{r+1}^{2}$ is a sheaf of modules over the fine sheaf $C^{\infty}$. Then since the segments

$$
H^{1}\left(X, \mathbb{Q}_{r+1}^{z}\right) \stackrel{j_{n+1}}{\rightarrow} H^{1}\left(X, \mathbb{Q}_{r}^{z}\right) \rightarrow H^{1}\left(X, \mathbb{Q}_{r} / \mathbb{Q}_{r+1}^{z}\right)
$$

are exact (by Lemma 3.4) each $j_{r+1}$ is onto for $r=2, \ldots, n$, and so is the composite $j_{3} j_{4} \cdots j_{n}$. But $H^{1}\left(X, \mathbb{Q}_{n}^{z}\right)=0$. Thus $H^{1}\left(X, \mathbb{Q}_{2}^{z}\right)$ is trivial.

Proof OF LeMma 3.8. The trick in proving this lemma is in defining $\sigma_{z} \cdot \rho_{z}$ is defined in a similar manner, and the bottom row is exact by Lemma 3.4. Commutativity of the diagram and that $\sigma_{z}$ and $\rho_{z}$ are isomorphisms with the desired properties follow by direct calculation.

Defintion of $\sigma_{z}$. Suppose that $z$ is a cocycle with respect to the cover $\left\{V_{j}\right\}_{J}$. For any refinement $\left\{W_{h}\right\}_{H}$ of the cover $\left\{V_{j}\right\}_{J}$, pick a map $\alpha: H \rightarrow J$ such that $W_{h}<V_{\alpha(h)}$. In this proof write $z(i, j)$ for $z_{i j}$. Define a map $\sigma_{\alpha, 2}$ : $C^{1}\left(\left\{W_{h}\right\}_{H}, \mathbb{Q}\right) \rightarrow C^{1}\left(\left\{W_{h}\right\}_{H}, \mathbb{Q}^{z}\right)$ where $C^{1}\left(\left\{W_{h}\right\}_{H}, \mathbb{Q}\right)$ [resp., $\left.C^{1}\left(\left\{W_{h}\right\}_{H}, \mathbb{Q}^{z}\right)\right]$ is the set of 1-cochains with respect to the cover $\left\{W_{h}\right\}_{H}$ with coefficients in the sheaf $\mathbb{Q}$ [resp., $\mathbb{Q}^{2}$ ], by setting

$$
\sigma_{\alpha, z}: g=\{g(h, k)\} \mapsto\left\{(z(j, \alpha(h)) g(h, k) z(\alpha(k), j))_{J}\right\} .
$$

A direct check shows that $\sigma_{\alpha, z}$ sends cocycles to cocycles.

If $g=\{g(h, k)\}$ and $f=\{f(h, k)\}$ are cohomologous cocycles in $C^{1}\left(\left\{W_{h}\right\}_{H}, \mathbb{Q}\right)$, then there exists $d=\{d(h)\}$ in the set of 0-cochains $C^{0}\left(\left\{W_{h}\right\}_{H}, \mathbb{Q}\right)$ such that $g(h, k)=d(h)^{-1} f(h, k) d(k)$ for every $h, k$. Notice that $\left\{(z(j, \alpha(h)) d(h) z(\alpha(h), j))_{J}\right\}$ is in $C^{0}\left(\left\{W_{h}\right\}_{H}, \mathbb{Q}^{z}\right)$. This establishes the equivalence of $\sigma_{\alpha, z}(g)$ and $\sigma_{\alpha, z}(f)$.

Then $\sigma_{\alpha, z}$ induces a map on cohomology. It remains to show that the map induced on cohomology is independent of the choice of $\alpha$. If $\beta: H \rightarrow J$ is another map such that $W_{h}<V_{\beta(h)}$ and $g=\{g(h, k)\}$ is a cocycle in $C^{1}\left(\left\{W_{h}\right\}_{H}, \mathbb{Q}\right)$, then notice that the cochain $\left\{(z(j, \alpha(h)) z(\beta(h), j))_{J}\right\}$ is in $C^{0}\left(\left\{W_{h}\right\}_{H}, \mathbb{Q}^{2}\right)$ and establishes the equivalence of $\sigma_{\alpha, z}(g)$ and $\sigma_{\beta, z}(g)$.

Now define $\sigma_{z}: H^{1}(X, \mathbb{Q}) \rightarrow H^{1}\left(X, \mathbb{Q}^{z}\right)$ to be the map induced by $\sigma_{\alpha, z}$. Using $p z(i, j)$ for $z(i, j)$, a similar procedure applies to give $\rho_{z}: H^{1}\left(X, \mathbb{Q} / \mathscr{C}_{2}\right)$ $\rightarrow H^{1}\left(X, \mathbb{Q} / \mathbb{Q}_{2}^{z}\right)$. 
5. Proofs for the structure theorems for $\operatorname{Aut}\left(\Lambda R^{n}\right)$.

Notation. For $c=\Sigma \partial_{i} \otimes w_{i}$ in $C(U)$ write $\overline{\Sigma \partial_{i} w_{i}}$. Also write $\Sigma f_{i} v_{i}$ for the sum $\Sigma f_{i} \otimes v_{i}$ in $\Lambda R^{n}(U)$.

Proof OF THEOREM 2.3. Notice that $e(U)<\operatorname{Der}\left(\Lambda R^{n}(U)\right)$, the set of derivations of $\Lambda R^{n}(U)$, since if $c=\sum \partial_{i} w_{i}$ is in $e(U)$, the linear map

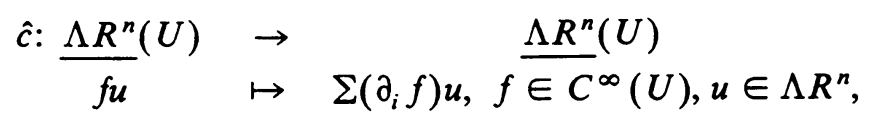

is a derivation. Moreover, $\hat{c}^{n}=0$ for all $c$ in $\mathcal{C}(U)$, and $\tilde{c}=\exp (\hat{c})$ is a well-defined algebra automorphism of $\Lambda R^{n}(U)$. Since $C$ is a sheaf, the restriction of $c$ to any open set $V$ in $U$ makes $\tilde{c}$ a map of sheaves.

If $d=(R, S)$ in $\mathscr{Q}(U)$, we can regard $d$ as a linear map $d: \Lambda^{1} R^{n} \rightarrow \Lambda R^{n}(U)$ where $d(x)=R(x)+S(x)$ for $x \in \Lambda^{1} R^{n}$. This map extends uniquely to a map of algebras $\hat{d}: \Lambda R^{n} \rightarrow \Lambda R^{n}(U)$. Then $\hat{d}$ extends uniquely to a map of algebras, which is also a map of $C^{\infty}(U)$ modules $\tilde{d}: \Lambda R^{n}(U) \rightarrow \Lambda R^{n}(U) . \tilde{d}$ is in fact a bijection since we can construct an inverse for $\hat{d}$ as follows. Write $S=S_{3}+S_{5}+\cdots+S_{m}$ where $S_{j}$ is in $\operatorname{Hom}\left(R^{n}, C^{\infty}(U) \otimes \Lambda^{j} R^{n}\right)$. Then the composite

$$
\left(I, R^{-1} S_{3} \cdots S_{m}\right) \circ \cdots \circ\left(I, R^{-1} S_{4} S_{3}\right) \circ\left(I, R^{-1} S_{3}\right) \circ\left(R^{-1}, 0\right)
$$

is a left inverse for $\hat{d}$. Since $\mathscr{D}$ is a sheaf the restriction of $d$ to any open set $V$ in $U$ makes $\tilde{d}$ a map of sheaves.

Now define $\Phi(c, d)_{V}: \Lambda R^{n}(V) \rightarrow \Lambda R^{n}(V)$ where $\Phi(c, d)_{V}(f u)=\tilde{c}(f) \tilde{d}(u)$

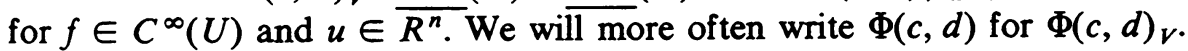
$\Phi(c, d)$ is clearly a map of sheaves of vector spaces.

We can express $\Phi(c, d)$ as a composition of algebra automorphisms as follows. Define an action of $\mathscr{D}(U)$ on $C(U)$ by setting $d \cdot\left(\Sigma \partial_{i} w_{i}\right)=\Sigma \partial_{i} \hat{d} w_{i}$ regarding $\operatorname{Der}(U)$ as a two-sided $C^{\infty}(U)$ module under the usual action. Since $\tilde{d}$ is an algebra automorphism we can define $d^{-1} \cdot\left(\Sigma \partial_{i} w_{i}\right)$ to mean $\sum \partial_{i} \tilde{d}^{-1} w_{i}$. Then observe that

$$
\Phi(c, d)=d \circ\left(d^{-1} \cdot c\right) .
$$

$\Phi$ is a bijection. Injectivity of $\Phi$ is trivial. For surjectivity of $\Phi$, let $\tau$ be in $\operatorname{Aut}\left(\Lambda R^{n}\right)(U)$. Since $\tau$ is an algebra automorphosm of $\Lambda R^{n}(U)$ it is sufficient to show that for some $c$ in $C(U)$ and all $f$ in $C^{\infty}(U)$ that $\tau(f)=\tilde{c}(f)$, and similarly that for some $d$ in $\mathscr{D}(U)$ and all $u$ in $\Lambda^{1} R^{n}, \tau(u)=\tilde{d}(u)$.

$\tau(f)=\tilde{c}(f)$ for some $c$ in $\mathcal{C}(U)$. We will show inductively that $\tau(f)=\tilde{c}_{r}(f)$ modulo terms of exterior degree $>r$ for an appropriate choice of $c_{r}$.

Let $c_{0}=0 . \tau$ is an algebra automorphism, and thus preserves the nil radical, and the following diagram commutes 


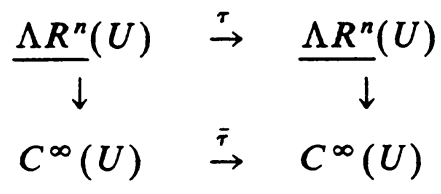

Since $\tau$ is an isomorphism of sheaves, so is $\bar{\tau}$. But then $\bar{\tau}$ must be the identity: Consider the dual picture. For an algebra $A$ let $A^{*}=\{f \in \operatorname{Hom}(A, R): \operatorname{ker} f$ contains an ideal of finite codimension $\}$. In our case, $\bar{\tau}$ induces a map $\bar{\tau}^{*}$ : $C^{\infty}(U)^{*} \rightarrow C^{\infty}(U)^{*}$. Notice that $U<C^{\infty}(U)^{*}$. Then if $u$ is in $U$ and $\bar{\tau}^{*}(u)=v \neq u$, pick an open set $V$ in $U$ such that $u$ is in $V$ and $v$ is not in $V$. However the following diagram must also commute since $\bar{\tau}$ is a map of sheaves:

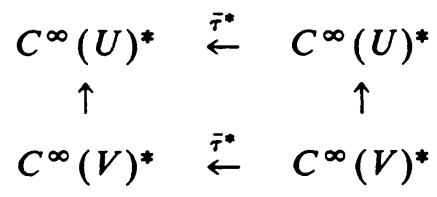

Therefore $\bar{\tau}^{*}(u)=v$ is in $V$, a contradiction. Then for $f$ in $C^{\infty}(U), \bar{\tau}(f)=$ $f\left(\bar{\tau}^{*}(u)\right)=f(u)$ for all $u$ in $U$; thus $\tau(f)=f$ plus terms of higher degree. This completes the base for induction.

Now assume that $\tau(f)-\tilde{c}_{r}(f)$ is in $\Lambda R^{n}(e, 2 r)(U)$ for all $f$ in $C^{\infty}(U)$ and

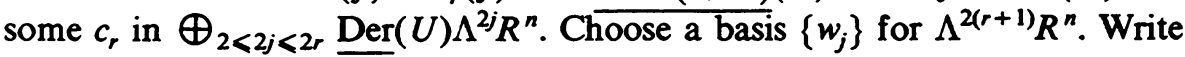
$c$ for $c_{r}$. Then

$$
(\tau-\tilde{c}) f=\sum \sigma_{j}(f) w_{j}+\text { terms of higher degree }
$$

where $\sigma_{j}$ is a linear endomorphism of $C^{\infty}(U)$. Also

$$
\begin{aligned}
(\tau-\tilde{c})(f g) & =\tau(f) \tau(g)-\tilde{c}(f) \tilde{c}(g) \\
& =\tau(f) \tau(g)-\tilde{c}(f) \tau(g)+\tilde{c}(f) \tau(g)-\tilde{c}(f) \tilde{c}(g) \\
& =(\tau(f)-\tilde{c}(f)) \tau(g)+\tilde{c}(f)(\tau(g)-\tilde{c}(g)) \\
& =\Sigma\left(g \sigma_{j}(f) w_{j}+f \sigma_{j}(g) w_{j}\right)+\text { higher terms. }
\end{aligned}
$$

But also

$$
(\tau-\tilde{c})(f g)=\Sigma \sigma_{j}(f g) w_{j}+\text { higher terms. }
$$

Using this it can be shown that each $\sigma_{j}$ is a derivation. Also $(\tau-(\tilde{c}+$ $\left.\left.\sum \sigma_{j} w_{j}\right)\right)(f)$ is in $\Lambda R^{n}(e, 2(r+1))(U)$, and $c+\sum \sigma_{j} w_{j}$ is in $\bigoplus_{2 \leqslant 2 j<2(r+1)} \operatorname{Der}(U) \otimes \Lambda^{2 j} R^{n}$, completing the inductive step.

$\tau(u)=\tilde{d}(u)$ for some $d$ in $\mathscr{D}(U)$ and all $u$ in $R^{n}$. Since $\tau$ respects the $Z_{2}$ grading $\tau\left(R^{n}\right)<\Lambda R^{n}(0,1)(U)$. Use the decomposition

$$
\underline{\Lambda R^{n}(0,1)(U)}=C^{\infty}(U) \otimes R^{n} \oplus \underline{\Lambda R^{n}(0,3)}(U)
$$

to write $\tau$ restricted to $R^{n}$ as a sum $A+B$ with $A$ in $\operatorname{Hom}\left(R^{n}, C^{\infty}(U) \otimes\right.$ $\left.R^{n}\right)$, and $B$ in $\operatorname{Hom}\left(R^{n}, \Lambda R^{n}(0,3)(U)\right)$. Then once we show that $A$ is 
invertible, setting $d=(A, B)$ we have $\tau(u)=\tilde{d}(u)$.

Let $\tilde{A}: \Lambda R^{n}(U) \rightarrow \Lambda R^{n}(U)$ be the algebra map generated by $A$. Denote the projection $\underline{\Lambda R^{n}}(U) \rightarrow C^{\infty}(U) \otimes R^{n}$ by $\pi$. The following diagram commutes

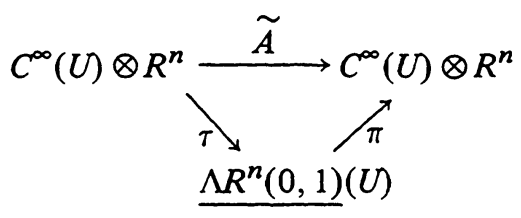

Notice that the composite $\tilde{A}=\pi \tau$ is a map of $C^{\infty}(U)$ modules. Since $\tau$ is an epimorphism, and $C^{\infty}(U) \otimes R^{n}$ is a set of generators for $\Lambda R^{n}(U)$ and also contains the indecomposables of $\Lambda R^{n}(U), A$ must be a surjective map of $C^{\infty}(U)$ modules. But a surjective map of free $C^{\infty}(U)$ modules of the same rank must be an isomorphism. Thus $A$ must be invertible.

Proof of THEOREM 2.5. (a) Let $a$ be in $Q_{i}(U)$, let $b$ be in $Q(U)$. Then if $x$ is in $\Lambda R^{n}(U), b^{-1} a b(x)-x=b^{-1}(a b(x)-b(x))$ is in $\Lambda R^{n}(i)(U)$ since $a(b(x))-b(x)$ is in $\Lambda R^{n}(i)(U)$ and $b^{-1}$ preserves $\Lambda R^{n}(i) \overline{(U)}$.

(b) For $a$ in $Q(U)$ and $f$ in $C^{\infty}(U)$, af $=f$ plus terms of higher degree. Thus $\mathbb{Q}(U)=\mathbb{Q}_{0}(U)=\mathbb{Q}_{1}(U)$. Notice that

$$
\Phi(c, d)(f)-f=\sum_{i=1}^{n} \frac{\hat{c}^{i}}{i !} f
$$

is in $\Lambda R^{n}(i)(U)$ if and only if $c$ is in $\operatorname{Der}(U) \otimes \Lambda R^{n}(e, i)$. Also if $d=(R, S)$ and $\bar{x}$ is in $R^{n}$, then $\Phi(c, d)(x)-x=\overline{R x}+S x-x$ is in $\Lambda R^{n}(i)(U)$ exactly when $R$ is the identity and $S$ is in $\operatorname{Hom}\left(R^{n}, \Lambda R^{n}(o, i)(U)\right)$.

(c) $i$ even. The crucial fact here is that $\left(c, \overline{d) \text { and }\left(c^{\prime}\right.}, d^{\prime}\right)$ in $Q_{i}(U)$ represent the same coset in $\mathbb{Q}_{i} / Q_{i+1}$ if and only if the components of $c$ and $c^{\prime}$ in $\operatorname{Der}(U) \otimes \Lambda^{i} R^{n}$ are the same. Then there is an obvious choice of representatives in $Q_{i}(U)$ and direct calculation shows that the group structure in $Q_{i} / Q_{i+1}(U)$ is sent to the additive structure in $\operatorname{Der}(U) \otimes \Lambda^{i} R^{n}$.

$i$ odd. Again, notice that $(c, d)$ and $\left(c^{\prime}, d^{\prime}\right)$ in $Q_{i}(U)$ represent the same class in $Q_{i} / Q_{i+1}$ exactly when $d$ and $d^{\prime}$ have the same component in $\operatorname{Hom}\left(R^{n}, C^{\infty}(U) \otimes \Lambda^{i} R^{n}\right)$.

(d) The $C^{\infty}(U)$ module structure in $\operatorname{Der}(U)$ gives $C(U)=\operatorname{Der}(U) \otimes$ $\Lambda R^{n}(e, 2)$ a $C^{\infty}(U)$ module structure. Define a $C^{\infty}(U)$ module structure on $\mathscr{Q}(U)$ by setting $f(R, S)=(R, f S)$ for $(R, S)$ in $\mathscr{D}(U)$ and $f$ in $C^{\infty}(U)$. The action of $C^{\infty}(U)$ on $Q(U)=C(U) \times \mathscr{D}(U)$ is the usual action on a product. Notice that $f: \mathbb{Q}(U) \rightarrow \mathbb{Q}(U)$ is not necessarily a group homomorphism for $f$ in $C^{\infty}(U)$, but on the quotients $f: \mathbb{Q}_{i} / \mathbb{Q}_{i+1}(U) \rightarrow \mathbb{Q}_{i} / \mathbb{Q}_{i+1}(U)$ is a map of groups.

The hard part is to show that if $z$ is in $\mathbb{Q}(U)$, the automorphism of $\mathbb{Q}_{i} / \mathbb{Q}_{i+1}$ 
induced by conjugation by $z$ is a map of $C^{\infty}(U)$ modules. The proof is by direct calculation. Write $z=(c, d)$. Then $\Phi(c, d)=d \circ\left(d^{-1} \cdot c\right)$. Notice that conjugation by any element of $e(U)$ induces the identity on $\mathbb{Q}_{i} / \mathbb{Q}_{i+1}$, so that, if $a$ is in $Q_{i} / Q_{i+1}(U)$,

$$
z a z^{-1}=d \circ\left(d^{-1} \cdot c\right) \circ a \circ\left(d^{-1} \cdot c\right)^{-1} \circ d^{-1}=d \circ a \circ d^{-1} .
$$

If $i$ is even, represent $a$ by $b$ in $\underline{\operatorname{Der}}(U) \otimes \Lambda^{i} R^{n}$. Then for $f, g$ in $C^{\infty}(U)$,

$$
d \circ f b \circ d^{-1}(g)=d \circ f b(g)=d \cdot f b(g) \text {. }
$$

Also, $f\left(d \circ b \circ d^{-1}\right)(g)=f d \circ b(g)=f d \cdot b(g)$. But $d \cdot f b$ and $f d \cdot b$ represent the same class in $\mathbb{Q}_{i} / \mathbb{Q}_{i+1}(U)$ by the remark in part (c) and hence $z \circ f b \circ z^{-1}$ and $f z \circ b \circ z^{-1}$ represent the same class in $Q_{i} / Q_{i+1}(U)$.

For $i$ odd, represent $a$ by $q$ in $\operatorname{Hom}\left(R^{n}, C^{\infty}(U) \otimes \Lambda^{i} R^{n}\right)$ and notice that $d \circ f q \circ d^{-1}=f d \circ q \circ d^{-1}$ since $d$ is a homomorphism of $\Lambda R^{n}(U)$ as $C^{\infty}(U)$ modules. This completes the proof of part (d).

\section{REFERENCES}

1. F. Berezin, The method of second quantization, Academic Press, New York, 1966.

2. F. Berezin and G. Kac, Lie groups with commuting and anticommuting parameters, Math. USSR-Sb. 11 (1970), 311-326.

3. F. Berezin and D. Leites, Supervarieties, Soviet Math. Dokl. 16 (1975), 1218-1222.

4. L. Corwin, Y. Ne'eman and S. Sternberg, Graded Lie algebras in mathematics and physics (Bose-Fermi symmetry), Rev. Modern Phys. 47 (1975), 573-604.

5. J. Dell and L. Smolin, A differential-geometric formulation of supersymmetry, Harvard Preprint (in preparation).

6. F. Hirzebruch, Topological methods in algebraic geometry, Springer-Verlag, New York, 1965.

7. V. Kac, Lie superalgebras, Advances in Math. 26 (1977), 8-96.

8. B. Kostant, Graded manifolds, graded Lie theory and prequantization. Differential geometric methods in mathematical physics, Lecture Notes in Math., vol. 570, Springer-Verlag, Berlin, 1977, pp. 177-306.

Department of Mathematics, Massachusetts institute of Technology, Cambridge, MASSACHUSETTS 02139

Current address: Department of Pure Mathematics and Mathematical Statistics, Cambridge University, 16 Mill Lane, Cambridge CB2 1SB, England 